\title{
Trabalho noturno e alterações de peso corporal autopercebidas pelos profissionais de enfermagem
}

\author{
Night work and self-perceived body weight changes among nursing professionals \\ Trabajo nocturno y alteraciones en el peso corporal según los trabajadores de enfermería
}

\author{
Maria Yvone Chaves Mauro', Alcione Morais dos Santos Rebelo", Alexandra de Oliveira Matias Ferreira"II, \\ Nathalia Pey Tournillon Sper ${ }^{\prime V}$, Maria Isabel Silva Santos ${ }^{V}$, Cristiane Helena Gallasch ${ }^{v i}$
}

\begin{abstract}
RESUMO
Objetivo: avaliar as alterações de peso corporal em trabalhadores de enfermagem do turno noturno. Metodologia: estudo quantitativo, exploratório, descritivo, realizado em um hospital federal de grande porte do Rio de Janeiro. Foram estudadas variáveis sóciodemográficas; influências do turno noturno sobre o organismo e índice de massa corporal, após aprovação do Comitê de Ética em Pesquisa da instituição. Resultados: os 89 trabalhadores de enfermagem apresentaram ganho de peso médio de aproximadamente $20 \mathrm{Kg}$ a partir da admissão no turno noturno, sendo que os enfermeiros referiram maior influência da ausência de sono sobre o organismo, e maior exaustão quando comparados aos dados das demais categorias. Conclusão: considerando as desordens hormonais e os impactos sociais do serviço noturno, é imprescindível a implementação de mudanças para uma cultura prevencionista, seja por programas institucionais ou pesquisas intervencionistas, capazes de desenvolver medidas que conduzam ao autorreconhecimento e à promoção do bem-estar físico, mental e social dos trabalhadores de enfermagem.

Descritores: Trabalho noturno; assistência noturna; promoção da saúde; saúde do trabalhador.
\end{abstract}

\begin{abstract}
Objective: to evaluate body weight changes in nursing workers on the night shift. Methodology: this quantitative, exploratory, descriptive study, conducted at a large federal hospital in Rio de Janeiro, after approval of the institution's research ethics committee, considered socio-demographic variables, influence of the night work on the organism, and body mass index. Results: average weight gain among the 89 nursing workers was approximately $20 \mathrm{~kg}$ since admission to night work, and nurses reported greater influence of lack of sleep on the body, and greater exhaustion as compared with data on the other categories. Conclusion: considering the hormonal disorders and social impacts of night work, it is essential to implement changes towards a culture of prevention, through either institutional programs or interventionist research able to develop measures that lead to self-recognition and promotion of nursing workers' physical, mental and social wellbeing.

Descriptors: Night work, night care, health promotion, occupational health.

\section{RESUMEN}

Objetivo: evaluar las alteraciones de peso corporal en trabajadores de enfermería del turno nocturno. Metodología: estudio cuantitativo, exploratorio, descriptivo, realizado en un gran hospital federal en Río de Janeiro. Se estudiaron las variables sociodemográficas, la influencia reportada de la guardia nocturna en el organismo y el índice de masa corporal, tras la aprobación del Comité de Ética de Investigación de la Institución. Resultados: Los 89 trabajadores de enfermería tuvieron un aumento de peso promedio de aproximadamente $20 \mathrm{~kg}$ desde el ingreso en el turno nocturno, y los enfermeros informaron una mayor influencia de la falta de horas dormidas sobre el cuerpo y un mayor agotamiento en comparación con los datos de las otras categorías. Conclusión: Teniendo en cuenta los trastornos hormonales y los impactos sociales del servicio nocturno, es esencial implementar cambios hacia una cultura de prevención, ya sea a través de programas institucionales o de investigación intervencionista, capaces de desarrollar medidas que conduzcan al auto reconocimiento y a la promoción del bienestar físico, mentales y social de los trabajadores de enfermería.

Descriptores: Trabajo nocturno, cuidados nocturnos, promoción de la salud, salud laboral.
\end{abstract}

\section{INTRODUÇÃO}

Sabe-se que, no sistema de saúde, o trabalho por turnos é considerado necessário e indispensável para garantir a continuidade dos cuidados durante as 24 horas do dia. Sendo o trabalho noturno, em particular, uma das razões mais frequentes para a interrupção dos ritmos circadianos, causando alterações significativas no sono e nas funções biológicas, observa-se impacto significativo sobre o bem-estar físico e psicológico dos trabalhadores, condicionando negativamente o desempenho no trabalho ${ }^{1-3}$.

'Enfermeira. Doutora em Enfermagem. Professora Titular Aposentada da Faculdade de Enfermagem da Universidade do Estado do Rio de Janeiro, Brasil. E-mail: mycmauro@uol.com.br. "Enfermeira. Mestre em Enfermagem pela Faculdade de Enfermagem da Universidade do Universidade do Estado do Rio de Janeiro, Brasil. E-mail: alcionedejesus@bol.com.br. I'Enfermeira. Doutoranda em Enfermagem pela Escola de Enfermagem Aurora de Afonso Costa da Universidade Federal Fluminense, Niterói, Brasil. E-mail: alexandrauff@gmail.com. NEnfermeira. Mestranda em Enfermagem pela Faculdade de Enfermagem da Universidade do Estado do Rio de Janeiro, Brasil. E-mail: nathy.sper@globo.com.

Enfermeira. Mestranda em Enfermagem pela Faculdade de Enfermagem da Universidade do Estado do Rio de Janeiro, Brasil. E-mail: mariaisabel2610@hotmail.com.

viEnfermeira. Doutora em Enfermagem. Professora Adjunta da Faculdade de Enfermagem da Universidade do Estado do Rio de Janeiro, Brasil. E-mail: cristiane.gallasch@gmail.com. V"Estudo desenvolvido a partir do macroprojeto Inovação de gestão das condições de trabalho em saúde para profissionais do Sistema Único de Saúde - SUS/Brasil, financiado pelo Conselho Nacional de Desenvolvimento Científico e Tecnológico (CNPq), que deu origem ao subprojeto Descanso noturno: influências da ergonomia na adaptação do trabalho de enfermagem 
Existem diferentes níveis de adaptação e tolerância ao trabalho noturno, que podem causar alterações de saúde, em maior ou menor intensidade, em variados intervalos de tempo. As repercussões das diversas influências do turno noturno podem variar amplamente entre os trabalhadores, relacionando-se a fatores individuais, como idade, traços de personalidade e características fisiológicas ${ }^{4-5}$. Além disso, a adaptação ao trabalho, incluindo o horário de trabalho, e as condições sociais, como número de empregos, idade dos filhos, moradia, tempo e forma de deslocamento também são determinantes para possíveis alterações psicofisiológicas 4 .

Os trabalhadores do turno noturno são propensos à privação do sono, desalinhamento dos ritmos circadianos, com consequente sonolência e déficits de desempenho relacionados ao sono ${ }^{5}$. O horário noturno é a fase em que se desativam ou atenuam mecanismos e sistemas vitais, com vistas à prevenção da exaustão. É, também, o momento em que são executados processos de recuperação e compensação de desgastes energéticos ou bioquímicos, surgidos no período de atividade. Quando a privação do sono é excessiva e persistente, pode haver desequilíbrio da estrutura do ritmo circadiano, com ocorrência de mal-estar, fadiga, diminuição do nível de alerta, sonolência, insônia, irritabilidade, prejuízo da agilidade mental, desempenho e eficiência, contribuindo para o erro durante o cuidado ao paciente ${ }^{4,6,7}$.

Assim, para garantir a segurança do paciente e do trabalhador, especialmente nos turnos noturnos, deve-se articular a implementação de intervalos para descanso, em locais apropriados, a fim de elevar o potencial de recuperação durante ou após longos turnos de trabalho. Há evidência de mais chances de recuperação após o trabalho para enfermeiros que dormem até entre 2,1 e 3 horas durante o plantão noturno, quando comparados aos enfermeiros que não dormem ${ }^{8}$.

Além de questões relacionadas à segurança na assistência, o ganho de peso ponderal tem sido relatado como maior entre os trabalhadores do turno noturno, elevando os riscos para doenças cardiovasculares, metabólicas e câncer $^{9-11}$.

A variação do peso noturno pode estar relacionada com a idade e com os anos de trabalho na profissão ${ }^{12}$. Prejuízos na dinâmica do sono podem também relacionar-se com a alta prevalência de sobrepeso e obesidade. Estudos com animais e com seres humanos indicam que a mudança do padrão de sono noturno pode influenciar de modo negativo a ingestão alimentar e, consequentemente, o estado nutricional ${ }^{13,14}$.

Assim, esse estudoVII justifica-se na ótica da ergonomia, considerando a necessidade de identificação de melhoria das condições laborais no ambiente de trabalho ${ }^{15}$, ou seja, de adaptação ergonômica do trabalho de enfermagem no turno noturno, considerando as que as diferenças nas atribuições no contexto hospitalar podem determinar níveis diferenciados de adaptação ao serviço noturno. Neste contexto, este estudo teve como objetivo avaliar as alterações de peso corporal em trabalhadores de enfermagem do turno noturno.

\section{MÉTODO}

Estudo exploratório, descritivo, com abordagem quantitativa, realizado com profissionais de enfermagem de um hospital federal de grande porte, de alta complexidade, no município do Rio de Janeiro. Optou-se pelo cálculo amostral probabilístico, com amostra finita ${ }^{16}$, ou seja, com tamanho $(n)$ maior ou igual a $5 \%$ do total da população de profissionais de enfermagem $(n=443)$. A partir da avaliação por estatístico independente, com intervalo de confiança de $95 \%$, estipulou a participação de, no mínimo, 89 trabalhadores de enfermagem.

Foram incluídos profissionais de todas as categorias de enfermagem, que aceitaram participar voluntariamente e eram vinculados à escala de plantões noturnos. Foram excluídos aqueles que estivessem em qualquer tipo de licença ou férias no momento da coleta de dados.

A coleta de dados foi realizada entre agosto e setembro de 2016, a partir de um formulário autoaplicado, elaborado pelos pesquisadores, baseado em pesquisa anterior relacionada à temática ${ }^{17}$. A partir da percepção do próprio participante do estudo, estruturado com perguntas abertas e fechadas, obteve-se informações sobre dados demográficos, formação, renda, tempo de serviço, jornada de trabalho, dependentes econômicos, peso autorreferido e influências do turno noturno no organismo. As medidas de peso e altura foram auto referidas, sendo calculado, posteriormente, o índice de massa corporal (IMC). O ponto de corte para obesidade foi $\geq 30 \mathrm{~kg} / \mathrm{m}^{2}$; sobrepeso 25 a 30 $\mathrm{kg} / \mathrm{m}^{2}$ e normal entre 20 a $25 \mathrm{~kg} / \mathrm{m}^{2}$.

Os dados foram tabulados no software Microsoft Excel ${ }^{\circ}$ e analisados com auxílio do Statistical Package for Social Science for Windows (SPSS ${ }^{\oplus}$ ). As variáveis sociodemográficas e informações antropométricas foram apresentados por meio de estatística descritiva. Para as variáveis nominais apresentam-se frequências absolutas e relativas. A avaliação da variação de peso foi analisada por meio do teste de variância (ANOVA), com formação de três grupos de análise, sem padrão de normalidade dos resultados. 
As associações entre alterações do peso corporal e o tempo de turno noturno, e a idade foram analisadas a partir de estatística inferencial, por meio do teste Qui-Quadrado - Pearson, um teste não paramétrico, destinado a encontrar um valor da dispersão para duas variáveis qualitativas, avaliando se as proporções observadas destes eventos apontam ou não diferenças significativas ou se diferem significativamente quanto à proporção destes acontecimentos, verificando a associação entre elas ${ }^{18}$. Considerou-se intervalo de confiança de $95 \%$, com p-valor $<0.05$ para assumir a hipótese de que não houve associação entre as variáveis estudadas.

O projeto de pesquisa obteve parecer favorável do Comitê de Ética em Pesquisa da instituição, sob protocolo número 066/2012, obedecendo aos requisitos da Resolução 466/12 do Conselho Nacional de Saúde.

\section{RESULTADOS}

A partir do cálculo amostral, foram distribuídos 150 formulários entre as diversas unidades de internação, para preenchimento por trabalhadores de enfermagem do turno noturno. Destes, 95 foram devolvidos, porém seis excluídos por preenchimento incompleto ou inadequado. O perfil dos participantes, obtido a partir dos dados sociodemográficos, laborais e educacionais, é demonstrado na Tabela 1.

Entre os participantes, 36 eram enfermeiros (40,45\%), 19 técnicos (21,35\%) e 34 auxiliares de enfermagem $(38,20 \%)$. A média de idade dos enfermeiros foi de $37,8( \pm 6,5)$ anos, de $43,8( \pm 8,1)$ para os técnicos e de $42,1( \pm 11)$ anos para os auxiliares de enfermagem. A declaração de estado civil casado é a mais frequente, presente em $61,80 \%$ da amostra, tendência seguida nas três categorias, assim como o sexo feminino $(73,5 \%)$. Em relação ao grau de escolaridade, o ensino médio tem maior frequência entre técnicos $(63,16 \%)$ e auxiliares de enfermagem (41,18\%), enquanto que os enfermeiros apresentaram pós-graduação completa em sua maioria (77,78\%).

Com relação ao vínculo empregatício, $58,43 \%$ do total dos profissionais tinham outro vínculo ( $n=52$ ). Porém, 59 outros turnos de trabalho foram identificados, o que leva ao entendimento de profissionais em três ou mais vínculos. Essa realidade reflete a uma renda líquida superior a $\mathrm{R} \$ 3001,00$ na maior parte dessa população.

Entre os profissionais, o enfermeiro apresenta o menor tempo (em anos) de trabalho na enfermagem $(13,7 \pm 7,3$ ), seguido dos técnicos de enfermagem $(15,1 \pm 6,4)$ e dos auxiliares de enfermagem $(17,4 \pm 9,4)$, sendo os auxiliares aqueles com mais tempo no turno noturno $(8,9 \pm 6,2$ anos). As horas dedicadas à profissão, referidas às duas últimas semanas trabalhadas foram maiores para os enfermeiros $(79,9 \pm 35,4$ horas).

$\mathrm{O}$ IMC anterior e posterior ao ingresso no trabalho noturno, calculado a partir de medidas antropométricas referidas, é demonstrado na Figura 1.

Todas as categorias apresentaram aumento do IMC médio, porém os enfermeiros apresentam maior variação numérica média $\left(24,7-29,7 \mathrm{~kg} / \mathrm{m}^{2}\right)$, passando da faixa normal para a de sobrepeso. Porém técnicos e auxiliares de enfermagem passaram da faixa de sobrepeso para obesidade.

A análise de variância (ANOVA) houve diferença entre as médias de diferença de peso no momento do estudo $(p=0,049)$. Houve variação média de $12,4 \mathrm{~kg}$, com mediana de $11 \mathrm{~kg}$, com 77 pessoas apresentando aumento (86,52\%), sete com perda $(7,86 \%)$ e cinco com estagnação de peso $(5,62 \%)$.

$\mathrm{O}$ teste de Qui-quadrado não demonstrou associação entre as variáveis: variação de peso $\mathrm{x}$ anos de trabalho noturno, variação de peso $\mathrm{x}$ idade, variação de peso $\mathrm{x}$ categoria profissional, variação de peso $\mathrm{x}$ horas de trabalho semanais $(p<0,05)$.

Ainda assim, a influência do turno noturno no controle do peso foi mais autorreferida pelos enfermeiros, que também referem dificuldade de relaxar após o trabalho, refletindo ao final do plantão em exaustão em $80 \%$ dos mesmos, seguidos pelos técnicos de enfermagem (70\%). Entre os técnicos de enfermagem, 47,4\% referiram conseguir relaxar apenas no segundo dia de folga. Os auxiliares de enfermagem sentem maior dificuldade de se concentrarem após o primeiro dia de folga (32,4\%) e apenas $47,1 \%$ sentem-se completamente recuperados após 8 horas de descanso. Os enfermeiros já apresentam seu desempenho prejudicado na parte final de seu dia de trabalho (38,9\%).

\section{DISCUSSÃO}

A enfermagem desenvolve suas atividades profissionais de cuidado integral em ambiente hospitalar durante 24 horas, em turnos ininterruptos para acompanhar indivíduos com dificuldades de saúde, tendo importância incontestável ${ }^{19}$.

A concentração de profissionais do sexo feminino era esperada, com é observado na prática e referido pela literatura $7,12,20-22$. Neste estudo, foi possível verificar que este perfil começa a ser desconstruído pela crescente concentração do sexo masculino, principalmente entre auxiliares de enfermagem $(26,5 \%)$. Isto pode explicar a maior concentração de homens no turno noturno se comparado ao diurno. Embora estudo anterior tenha apresentando 
menor frequência de homens na enfermagem, estes, quando presentes, estão alocados em sua maioria no turno noturno ${ }^{23}$.

Tabela 1: Dados sociodemográficos, laborais e educacionais de profissionais de enfermagem do turno noturno de um em hospital de grande porte. Rio de Janeiro, Brasil, 2016.

\begin{tabular}{|c|c|c|c|c|c|c|c|c|}
\hline \multirow[t]{2}{*}{ Variáveis } & \multicolumn{2}{|c|}{$\begin{array}{c}\text { Enfermeiro } \\
(n=36)\end{array}$} & \multicolumn{2}{|c|}{$\begin{array}{l}\text { Técnico } \\
(n=19)\end{array}$} & \multicolumn{2}{|c|}{$\begin{array}{c}\text { Auxiliar } \\
(n=34)\end{array}$} & \multicolumn{2}{|c|}{$\begin{array}{c}\text { Total } \\
(n=89)\end{array}$} \\
\hline & $n$ & $\%$ & $n$ & $\%$ & $\mathrm{n}$ & $\%$ & $n$ & $\%$ \\
\hline \multicolumn{9}{|l|}{ Sexo } \\
\hline Masculino & 4 & 11,11 & 4 & 21,05 & 9 & 26,47 & 17 & 19,10 \\
\hline Feminino & 32 & 88,89 & 15 & 78,95 & 25 & 73,53 & 72 & 80,90 \\
\hline \multicolumn{9}{|l|}{ Idade } \\
\hline $20-30$ & 1 & 2,79 & 0 & 0 & 5 & 14,70 & 6 & 6,74 \\
\hline $31-40$ & 25 & 69,44 & 7 & 36,84 & 15 & 44,12 & 47 & 52,81 \\
\hline $41-50$ & 7 & 19,44 & 8 & 42,11 & 7 & 20,59 & 22 & 24,72 \\
\hline $51-60$ & 3 & 8,33 & 4 & 21,05 & 7 & 20,59 & 14 & 15,73 \\
\hline \multicolumn{9}{|l|}{ Estado Civil } \\
\hline Casado & 25 & 69,44 & 10 & 52,63 & 20 & 58,82 & 55 & 61,80 \\
\hline Solteiro & 10 & 27,78 & 3 & 15,78 & 8 & 23,53 & 21 & 23,59 \\
\hline Viúvo & 1 & 2,78 & 2 & 10,53 & 2 & 5,89 & 5 & 5,62 \\
\hline Separado ou divorciado & 0 & 0 & 2 & 10,53 & 4 & 11,76 & 6 & 6,74 \\
\hline Indeterminado & 0 & 0 & 2 & 10,53 & 0 & 0 & 2 & 2,25 \\
\hline \multicolumn{9}{|l|}{ Grau de Escolaridade } \\
\hline Ensino Médio Completo & 0 & 0 & 12 & 63,16 & 14 & 41,18 & 26 & 29,21 \\
\hline Ensino superior completo & 8 & 22,22 & 4 & 21,05 & 10 & 29,41 & 22 & 24,72 \\
\hline $\begin{array}{l}\text { Pós-graduação } \\
\text { (Lato/Scrito Sensu) }\end{array}$ & 28 & 77,78 & 3 & 15,79 & 10 & 29,41 & 41 & 46,07 \\
\hline \multicolumn{9}{|l|}{$\begin{array}{l}\text { Outro vínculo } \\
\text { empregatício }\end{array}$} \\
\hline Sim & 20 & 55,56 & 12 & 63,16 & 20 & 58,82 & 52 & 58,43 \\
\hline Não & 16 & 44,44 & 7 & 36,84 & 14 & 41,18 & 37 & 44,57 \\
\hline \multicolumn{9}{|l|}{$\begin{array}{l}\text { Qual o turno do outro } \\
\text { vínculo }\end{array}$} \\
\hline Noturno & 7 & 35 & 3 & 25 & 3 & 15 & 13 & 25 \\
\hline Diurno & 13 & 65 & 9 & 75 & 8 & 40 & 30 & 57,7 \\
\hline Outros & 0 & 0 & 0 & 0 & 9 & 45 & 9 & 17,3 \\
\hline \multicolumn{9}{|l|}{$\begin{array}{l}\text { Renda Líquida } \\
\text { aproximada }\end{array}$} \\
\hline Entre 500 e 1000 & 0 & 0 & 1 & 5,26 & 3 & 8,82 & 4 & 4,49 \\
\hline Entre 1001 e 2000 & 0 & 0 & 2 & 10,52 & 2 & 5,88 & 4 & 4,49 \\
\hline Entre 2001 e 3000 & 13 & 36,11 & 2 & 10,52 & 4 & 11,76 & 19 & 21,35 \\
\hline Entre 3001 e 5000 & 8 & 22,22 & 14 & 68,44 & 16 & 47,07 & 38 & 42,69 \\
\hline Mais de 5000 & 14 & 41,67 & 1 & 5,26 & 9 & 26,47 & 24 & 26,98 \\
\hline $\begin{array}{l}\text { Anos de trabalho na } \\
\text { enfermagem (DP) }\end{array}$ & \multicolumn{2}{|c|}{$13,7( \pm 7,3)$} & \multicolumn{2}{|c|}{$15,1( \pm 6,4)$} & \multicolumn{2}{|c|}{$17,4( \pm 9,4)$} & & \\
\hline $\begin{array}{l}\text { Anos de trabalho no } \\
\text { turno noturno (DP) }\end{array}$ & \multicolumn{2}{|c|}{$8,9( \pm 6,2)$} & \multicolumn{2}{|c|}{$6,5( \pm 4,5)$} & \multicolumn{2}{|c|}{$12,9( \pm 6,9)$} & & \\
\hline $\begin{array}{c}\text { Horas semanais } \\
\text { dedicadas à profissão (DP) }\end{array}$ & \multicolumn{2}{|c|}{$79,9( \pm 35,4)$} & \multicolumn{2}{|c|}{$71,8( \pm 38,5)$} & \multicolumn{2}{|c|}{$66,4( \pm 30,2)$} & & \\
\hline
\end{tabular}

Legenda: $\mathrm{DP}=$ desvio-padrão 


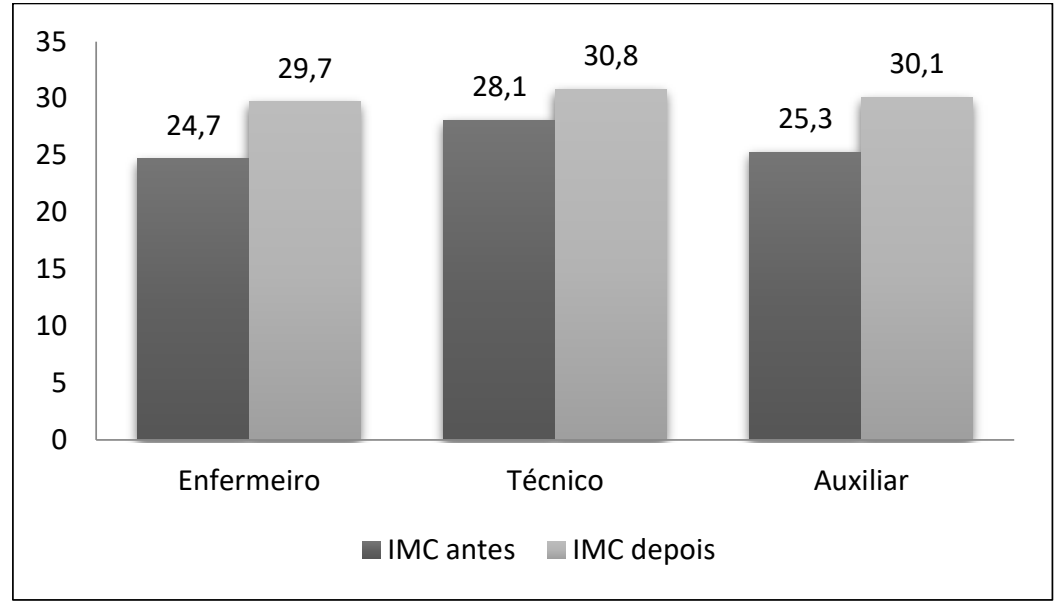

Figura 1: Comparação do Índice de Massa Corporal (IMC) antes e depois no ingresso do turno noturno de profissionais de enfermagem em hospital de grande porte. Rio de Janeiro, Brasil, 2016.

Com relação ao vínculo empregatício, as diferenças individuais na adaptação ao trabalho em turnos variam de acordo com cada indivíduo, e a exposição contínua a horários irregulares afeta a ritmicidade biológica, podendo ocorrer distúrbios do sono, humor, gastrointestinais e cardiovasculares ${ }^{3}$. 0 acúmulo de vínculo, não reflete, necessariamente, em melhor renda. Os enfermeiros apresentaram, em sua maioria, renda líquida menor que $\mathrm{R} \$ 5000$ reais. Estes resultados díspares também são encontrados na literatura, em que a faixa salarial prevalente está entre $R \$ 2.001,00$ a $\mathrm{R} \$ 3.000,00$, os quais podem ser considerados baixos para a carga horária exercida, com uma carga horária entre 21 a 40 horas semanais $^{21}$.

O aumento do peso corporal observado é semelhante a estudo anterior, onde o trabalho noturno foi associado a um ganho de peso maior que $0,24 \mathrm{~kg} / \mathrm{m}^{2}$, e, o diurno a $0,15 \mathrm{~kg} / \mathrm{m}^{212}$. Sabe-se que o maior o intervalo de tempo acordado, além de promover a alteração hormonal capaz de aumentar a ingestão calórica, pode possibilitar uma maior oportunidade para a ingestão alimentar ${ }^{24}$.

Estudos sugerem que a gordura corporal total pode estar associada com uma maior latência para o sono, contribuindo com a redução do tempo total de descanso. Este fato é muito comum principalmente onde os trabalhadores do turno noturno que não têm disponível sua refeição no próprio local; diante disto, os profissionais buscam alimentos de alta densidade energética para um turno que apresenta menor concentração de rotinas e gasto energético elevado ${ }^{25,26}$.

A sobrecarga de trabalho e escassez de recursos humanos de enfermagem, em conjunto, são também importantes aspectos que influenciam diretamente no aumento da sobrecarga e cansaço ${ }^{27}$. Em relação às influências do turno noturno no organismo dos trabalhadores, destaca-se que o horário de trabalho, assim como as dificuldades relacionadas ao descanso após a saída, pode levar a desajustes do ritmo circadiano, com aparecimento de alterações psicológicas, gastrointestinais, metabólicas, com ocorrência de obesidades e outras doenças crônicas ${ }^{28}$.

As repercussões sobre os aspectos sociais são mais percebidas pelos enfermeiros se comparado aos demais profissionais da enfermagem. Destaca-se que o enfermeiro do período noturno muitas vezes acumula funções direcionadas à supervisão, o além das atividades assistenciais mais complexas, fatores que podem aumentar as cargas físicas e mentais ${ }^{22}$.

Entretanto, em estudo realizado com trabalhadores de unidade de cuidados intensivos, o trabalho noturno não foi visualizado somente como causador de doenças e desequilíbrios ao seu bem-estar. Mesmo com relato de sobrecarga de trabalho, aspectos como maior disponibilidade de tempo, de atenção à família, à economia, à possibilidade de qualificação são elementos considerados satisfatórios a aqueles que trabalham neste período ${ }^{29}$.

Em relação aos aspectos percebidos em relação às condições de descanso no turno noturno, foi apontado em sua totalidade pelos profissionais de enfermagem que eles têm permissão de descansar pelo menos três horas. Os profissionais em sua maioria consideraram o local para o descanso adequado, fator incomum se comparar com o restante do país, onde não se observa esse tipo de condição para o descanso noturno. É relevante apontar que os profissionais que cochilam pelos menos duas horas no turno noturno aumentam a recuperação pós-trabalho ${ }^{8}$. 


\section{CONCLUSÃO}

Verificou-se aumento de IMC, por meio de dados de peso autorreferidos, entre os trabalhadores de todas as categorias de enfermagem, com enfermeiros passando da faixa normal de peso para a de sobrepeso; e técnicos e auxiliares de enfermagem de sobrepeso para obesidade. Além disso, houve diferença entre as médias de diferença de peso.

Destaca-se que o trabalhador do serviço noturno de enfermagem está sujeito às modificações endócrinas e processos adaptativos em decorrência da ausência de sono, havendo necessidade de promoção do descanso noturno de duas a três horas.

É imprescindível a implementação de mudanças para uma cultura prevencionista, seja por programas institucionais ou pesquisas intervencionistas, capazes de desenvolver medidas que conduzam ao autorreconhecimento das alterações corporais, além da promoção do bem-estar físico, mental e social dos trabalhadores de enfermagem, incluindo a alimentação e lazer, promoção do sono. Medidas de controle e vigilância sobre desordem das condições de alerta devem ser promovidas, além de ajustes mais efetivos para resguardar o ciclo dos ritmos biológicos, com base numa abordagem educacional, contemplando as rotinas dos trabalhadores que atendam às necessidades de eficácia da coordenação de enfermagem.

Estas ações resultarão na promoção do bem-estar, da saúde e da satisfação dos profissionais, assegurando, consequentemente, a qualidade dos cuidados, segurança dos pacientes e produtividade das atividades de enfermagem.

\section{REFERÊNCIAS}

1. Korompeli A, Muurlink O, Tzavara C, Velonakis E, Lemonidou C, Sourtzi P. Influence of shiftwork on Greek nursing personnel. Saf health work. 2014 [cited 2017 Jan 03]; 5(2):73-9. Doi: http://dx.doi.org/10.1016/j.shaw.2014.03.003

2. Costa G. Shift work and health: current problems and preventive actions. Saf health work. 2010 [cited 2017 Jan 03 ]; 1(2):112-23. Doi: http://dx.doi.org/10.5491/SHAW.2010.1.2.112

3. Costa G, Accattoli MP, Garbarino S, Magnavita N, Roscelli F. Sleep disorders and work: guidelines for health surveillance, risk management and prevention. Med lav. 2013 [cited 2017 Jan 03]; 104(4):251-266. Available from: https://www.ncbi.nlm.nih.gov/pubmed/24228304

4. Costa IMAR. Trabalho por turnos, saúde e capacidade para o trabalho dos enfermeiros. Dissertação de Mestrado apresentada à Faculdade de Medicina da Universidade de Coimbra, para a obtenção do grau de Mestre em Saúde Ocupacional. 2009.

5. Johnson AL, Jung L, Brown KC, Weaver MT, Richards KC. Sleep Deprivation and Error in Nurses who Work the Night Shift. J nurs admin. 2014 [cited 2017 Jan 03]; 44(1):17-22. Doi: http://dx.doi.org/10.1097/NNA.0000000000000016

6. Azambuja AAA, Dias FM, Bottcher LB. Os efeitos do trabalho noturno na saúde dos profissionais. Revista interdisciplinar encontro das ciências. 2019 [cited 2019 Apr 03]; 2(1):582-92. Available from: http://www.fvs.edu.br/riec/index.php/riec/article/view/25

7. Ruggiero JS, Redeker NS. Effects of napping on sleepiness and sleep-related performance deficits in night-shift workers: A systematic review. Biol Res Nurs. 2014 [cited 2017 Jan 03]; 16(2):134-42. Available from: https://www.ncbi.nlm.nih.gov/pubmed/23411360

8. Palermo TAC, Rotenberg L, ZeitouneRCG, Silva-Costa A, Souto EP, Griep RH. Napping during the night shift and recovery after work among hospital nurses. Rev latino-am enfermagem. 2015 [cited 2017 Jan 03]; 23(1):114-21. Doi: http://dx.doi.org/10.1590/0104-1169.0147.2532

9. Vetter C, Devore EE, Wegrzyn LR, Massa J, Speizer FE, Kawachi I, et al. Association between rotating night shift work and risk of coronary heart disease among women. JAMA. 2016 [cited 2017 Jan 03]; 315(16):1726-34. Doi: http://dx.doi.org/10.1001/jama.2016.4454

10. Schernhammer ES, Feskanich D, Liang G, Han J. Rotating night-shift work and lung cancer risk among female nurses in the United States. J Epidemiol. 2013 [cited 2017 Jan 06]; 178(9):1434-44. Doi: http://dx.doi.org/10.1093/aje/kwt155

11. Wang F, Zhang L, Zhang Y, Zhang B, He Y, Xie S, et al. Meta-analysis on night shift work and risk of metabolic syndrome. Obesity reviews. 2014 [cited 2017 Jan 06]; 5(9):709-20. Doi: http://dx.doi.org/10.1111/obr.12194

12. Marqueze EC, Lemos LC,Soares N,Lorenzi-Filho G, Moreno CRC. Weight gain in relation to night work among nurses. Work. 2012 [cited 2017 Jan 03]; 41(Sup1):2043-204. Doi: http://dx.doi.org/10.3233/WOR-2012-0429-2043

13. Coelho MP, Pinto OO, Motal MC, Crispim CA. Prejuízos nutricionais e distúrbios no padrão de sono de trabalhadores da Enfermagem. Rev bras enferm. 2014 [cited 2017 Jan 03]; 67(5):832-42. Doi: http://dx.doi.org/10.1590/0034-7167.2014670523

14. Marti AR, Meerlo P, Grønli J, van Hasselt SJ, Mrdalj J, Pallesen S, et al. Shift in food intake and changes in metabolic regulation and gene expression during simulated night-shift work: a rat model. Nutrients. 2016 [cited 2017 Jan 03]; 8(11):712. Doi: http://dx.doi.org/10.3390/nu8110712

15. Silva LA, Secco IAO, Dalri RCMB, Araújo AS, Romano CC, Silveira SE. Ergonomics and occupational health nursing: prevention of health disorders. Rev enferm UERJ. 2011 [cited 2017 Jan 03]; 19(2):317-23. Available from: http://www.facenf.uerj.br/v19n2/v19n2a24.pdf

16. Ytriola MF. Introdução à Estatística. 7a. Ed. Rio de Janeiro: LTC, 1999. 
17. Costa A, Fischer F. Cochilos durante o trabalho noturno, necessidade de recuperação após o trabalho e percepção da fadiga entre os profissionais de enfermagem. [dissertação de mestrado] São Paulo: Faculdade de Saúde Pública. 2010 p. 20-111

18. Pluye $\mathrm{P}$, Hong QH. Combining the power of stories and the power of numbers: mixed methods research and mixed studies reviews. Annu rev public health. 2014 [cited 2017 Jan 03]; 35:29-45. Doi: http://dx.doi.org/10.1146/annurev-publhealth032013-182440

19. Machado LSF, Rodrigues EP, Oliveira LMM, Laudano RCS, Nascimento SCL. Health problems reported by nursing workers in a public hospital of Bahia. Rev bras enferm. 2014 [cited 2017 Jan 03]; 67(5):684-91. Doi: http://dx.doi.org/10.1590/00347167.2014670503

20. Guimarães ALO, Felli VEA. Notification of health problems among nursing workers in university hospitals. Rev bras enferm. 2016 [cited 2017 Jan 03]; 69(3):507-14. Doi: http://dx.doi.org/10.1590/0034-7167.2016690313i

21. Silva RM, Beck CLC, Zeitoune RCG, Prestes FC, Tavares JP, Guerra ST. Meaning of work for night nurses of a university hospital: descriptive study. Online brazilian J Nursing. 2011 [cited 2017 Jan 03]; 10(3). Doi: https://doi.org/10.5935/1676-4285.20113433

22. Santos TCMM, Inocente NJ, Martino MMF. Work shifts: chronotype relations and quality sleep. Journal of Nursing UFPE on line. 2014 [cited 2016 Jan 6]; 8(10):3437-43. Available from: https://periodicos.ufpe.br/revistas/revistaenfermagem/article/view/10076

23. Griep RH, Fonseca MM, Melo ECP, Portela L F, Rotenberg L. Nurses of large public hospitals in Rio de Janeiro: socio demographic and work related characteristics. Rev bras enferm. 2013 [cited 2017 Jan 03]; 66(spe):151-57. Doi: http://dx.doi.org/10.1590/S0034-71672013000700019

24. Crispim CA, Dáttilo IZM, Padilha HG, Tufik S,Mello MT. Relação entre Sono e Obesidade: uma Revisão da Literatura. Arq bras endocrinol metab. 2007 [cited 2017 Jan 03]; 51(7):1041-9. Doi: http://dx.doi.org/10.1590/S0004-27302007000700004

25. Moratoya EE, Carvalhaes GC, Wander AE, Almeida LMMC. Changes in food consumption pattern in Brazil and around the world. Revista de política agrícola. 2013 [cited 2017 Jan 03]; 22(1):72-84. Available from: https://ainfo.cnptia.embrapa.br/digital/bitstream/item/86553/1/Mudancas-no-padrao-de-consumo-alimentar-no-Brasil-e-nomundo.pdf

26. Patel SR, Hu FB. Short sleep duration and weight gain: a systematic review. Obesity. 2008 [cited 2017 Jan 03]; 16(3):643-53. Doi: http://dx.doi.org/10.1038/oby.2007.118

27. Scherer MDA, Oliveira NA, Pires DEP, Trindade LL, Gonçalves ASR, VieiraM. Increased workloads for nurse technicians in primary health care in brazil. Trab. Educ. saúde. 2016 [cited 2018 Dec 20]; 14(Suppl 1):89-104. Doi: http://dx.doi.org/10.1590/19817746-sol00030

28. Grillo LP, Albuquerque NR, Vieira NC, Mezadri T, Lacerda LLV. Risk and protective factors for the development of chronic diseases in nurses. Revista de Enfermagem Referência. 2018 [cited 2018 Dec 20]; IV(18):63-72. Doi: https://doi.org/10.12707/RIV18007

29. Silveira M, Camponogara S, Beck CLC, Costa VZ, Dalmolin GL, Arboit EL. Night work and its meanings for intensive care unit nursing. Rev. enferm. Uerj. 2016 [cited 2017 Oct 20]; 24(6):e17416. Doi: https://doi.org/10.12957/reuerj.2016.17416 